\title{
Acción comunitaria frente al fenómeno del cambio climático, en el páramo de la región del Guavio, Cundinamarca, Colombia
}

\section{Community action against the phenomenon of climate change, in the páramo of the Guavio region, Cundinamarca, Colombia}

\author{
Acção comunitária contra o fenómeno das alterações \\ climáticas, no páramo da região Guavio, \\ Cundinamarca, Colômbia
}

\author{
Sonia Astrid Mendoza Velásquez¹, Juan Guillermo Cano Muñoz² \& Fabián Rojas Sánchez³ \\ 'Zootecnista, Pedagogía para el Desarrollo del aprendizaje Autónomo, Magister en Sociología. \\ ${ }^{2}$ Ingeniero de Agroecología, Magister en Desarrollo Sostenible y Medio Ambiente. Ingeniero en \\ Agroecología. Especialista en Planeación ambiental y gestión integral de los recursos naturales
}

'Escuela de ciencias Agrícolas Pecuarias y del Medio Ambiente -ECAPMA. Universidad Nacional Abierta y a Distancia -UNAD. Bogotá. Colombia. 2,3Proyecto Agroecología. Parque Científico de Innovación

Social - UNIMINUTO. Corporación Universitaria Minuto de Dios - UNIMINUTO . Bogotá. Colombia.

1'sonia.mendoza@unad.edu.co, 2jcano@uniminuto.edu, ${ }^{3}$ fabian.rojas@uniminuto.edu

\section{Resumen}

El municipio de Junín, Cundinamarca, Colombia, hace parte de la zona de amortiguamiento del Parque Nacional Natural Chingaza y se caracteriza por poseer una gran variedad de recursos naturales, abundante agua y bosques naturales; sin embargo, presenta un alto grado de contaminación del recurso hídrico y adicionalmente, se ha presentado extinción de la fauna y la flora silvestre, destrucción de los bosques por talas y quemas indiscriminadas. La presente investigación tiene el propósito de documentar el proceso y analizar los resultados de la aplicación de la Investigación Acción Participante IAP como estrategia para la acción comunitaria frente al fenómeno del cambio climático, con los campesinos de los centros poblados de Chuscales y Claraval, del municipio de Junín. EI IAP utilizo la Evaluación de Sistemas de Manejo incorporando Indicadores de Sustentabilidad (MESMIS) y la cartografía social para el diagnóstico. Se realizaron sesiones de reflexión y aprendizaje con la comunidad a partir de los resultados. Se concluye que el modelo de IAP permitió identificar los problemas relacionados con la forma tradicional de producir, reducir el uso de agrotóxicos en los cultivos tradicionales, además, se promovió el uso de la agroecología para la producción de alimentos, la creación de huertas comunitarias, arreglos silvopastoriles para la producción de forraje, producción de ganadería lechera, y la siembra de árboles para protección de fuentes de agua. Al mismo tiempo, se impulsó la conformación de la asociación de productores y comercializadores de productos orgánicos con utilización de recursos propios y apoyo gubernamental.

Palabras clave: acción comunitaria, Investigación Acción Participante, mitigación de variabilidad climática, producción agroecológica. 


\section{Abstract}

The municipality of Junín, Cundinamarca, Colombia, is part of the buffer zone of the Natural Chingaza National Park and is characterized by a variety of natural resources, abundant water and natural forests; however, it has a high degree of pollution within water resources and additionally, presented extinction of fauna and flora, destruction of forests by logging and indiscriminate burning. This research is intended to document the process, and analyze the results of the application of the participant action research (IAP) as a strategy for community action against the phenomenon of climate change, with peasants from the towns of Chuscales and Clairvaux, of the municipality of Junín. IAP uses the evaluation of management systems incorporating sustainability indicators (MESMIS) and the social mapping for the diagnosis. Learning and reflection sessions were conducted with the community as a result. It was concluded that the IAP model allowed to identify the problems associated with traditional ways of production, reduced the use of agrochemicals in traditional crops, in addition, the use of agroecology for food production, promoted the creation of community gardens, arrangements silvopastoral for the production of fodder, production of dairy farming, and the planting of trees for protection of water sources. At the same time, it promoted the formation of the association of producers and traders of organic products trought of the use of this own resources and of the government support.

Key-words: community action, participatory action research, mitigation of climate variability, agro-ecological production.

\section{Resumo}

A cidade de Junin, Cundinamarca, Colômbia, faz parte da zona de amortecimento de Chingaza parque nacional natural é caracterizada por uma variedade de recursos naturais, água abundante e florestas naturais; no entanto, apresenta um alto grau de contaminação dos recursos hídricos e, além disso, apresentou extinção da fauna e da flora selvagens, a destruição de florestas por derrubadas e queimadas indiscriminadas. Esta pesquisa tem como objetivo documentar o processo e analisar os resultados da implementação da pesquisa-ação participativa IAP como uma estratégia de acção comunitária em relação ao fenómeno das alterações climáticas, com os agricultores nas cidades de Chuscales e Claraval no município Junin. O IAP, fez uso de Sistemas de Gestão de Avaliação incorporando Indicadores de Sustentabilidade (MESMIS) e mapeamento social para o diagnóstico. Sessões de reflexão e aprendizagem foram realizadas com a comunidade. Com base nos resultados. Concluiuse que o modelo de IAP, identificou os problemas associados com a forma tradicional de produção, reduzir a utilização de pesticidas em culturas tradicionais; promover o uso de agro-ecologia para a produção alimentar, a criação de hortas comunitárias, arranjos silvipastoris para fabricação de ração, produção de pecuária leiteira, e plantio de árvores para proteger as fontes de água. Ao mesmo tempo, promoveu a formação da associação de produtores e comerciantes de produtos orgânicos usando seus próprios recursos e apoio do governo.

Palavras-chave: ação comunitária, pesquisaação participativa, mitigação da variabilidade climática, produção ecológica.

\section{Introducción}

El Parque Nacional Natural (PNN) Chingaza, ubicado en la provincia del Guavio, Cundinamarca, Colombia, tiene zonas de amortiguamiento de gran importancia para la captación de agua y el desarrollo de ciudades como Bogotá y los municipios circunvecinos. El municipio de Junín hace parte de dicha provincia y se caracteriza por poseer una gran variedad de recursos naturales, abundante agua y bosques naturales; sin embargo, presenta un alto grado de contaminación del recurso 
hídrico por el inadecuado manejo de los residuos sólidos, y líquidos, que en muchos casos son tóxicos. Adicionalmente, se ha presentado una afectación a las lagunas de la región, extinción de la fauna y la flora silvestre, destrucción de los bosques por las talas y las quemas indiscriminadas. Por otra parte, la producción agrícola representa el $70,5 \%$ de cultivos transitorios y el $16,6 \%$ cultivos permanentes, de los cuales $12,9 \%$ de cultivos anuales son de arveja, papa, maíz, caña panelera y mora. También, produce el $15.3 \%$ de la leche que se comercializa en el departamento de Cundinamarca (CCB, 2008).

Los campesinos de los centros poblados de Chuscales y Claraval del municipio de Junín, no están ajenos a los cambios climáticos que ha sufrido la región en los últimos años. Por esta razón, un equipo de investigadores de la Corporación Universitaria Minuto de Dios - UNIMINUTO- y la Secretaría de Ciencia Tecnología e Innovación (SCTel) de la Gobernación de Cundinamarca, actuaron como agentes externos a la comunidad con el propósito de obtener la información necesaria para diseñar una propuesta de acción comunitaria que aliente la participación, la concientización y el empoderamiento de los campesinos en el abordaje de las acciones que se requiere emprender frente a la problemática ambiental, y específicamente en el cambio climático, como una realidad que está afectando y transformando profundamente los aspectos naturales y sociales del país y del planeta en general.

Abordar la problemática de forma responsable implica preguntarse:

- ¿Cuál es el aporte que desde la comunidad puede hacerse, para mitigar el cambio climático, realidad que afecta la cotidianidad de las sociedades rurales y urbanas de la zona de amortiguamiento del PNN Chingaza?

- ¿Cómo generar acciones de hecho que apunten al mejoramiento de la comunidad rural que vive en las zonas de reservorios de agua?
- ¿Cuál es la metodología apropiada para llegar al interior de la comunidad y que ella misma genere ejercicios positivos en favor del cambio de actitud y en la mejora de las prácticas de agricultura y ganadería tradicionales?

\section{Agroecología, como pretexto para la comunicación y el entendimiento}

La Investigación Acción Participativa (IAP) y el Diagnóstico Rural Participativo (DRP) permiten la comunicación entre los habitantes de la zona de estudio, hacen un diálogo claro y abierto de los problemas que diariamente ven en la región, sin detenerse a hablar de ellos como diálogos importantes para la supervivencia y sobre las formas de producir teniendo como prioridad los problemas estructurales. Es así como la indagación saca a flote los más íntimos temores sobre las formas de actuar, la comunicación dirigida, permeada por la investigación, da lugar a diálogos sobre temas de la cotidianidad que no están explícitos en el quehacer cotidiano. Estos temas que en su mayoría pueden estar identificados o no, pero que no son tema de análisis crítico, y menos aún temas para la acción. De esa forma los investigadores juegan un papel importante en la escogencia del tema, en la identificación sistemática de los problemas, la localización en el terreno del problema y las acciones a emprender (Bordeau, 1998).

La crisis medioambiental y socioeconómica de la agricultura convencional a escala mundial ha originado el surgimiento de la agroecología como un enfoque teórico y metodológico, utilizando varias disciplinas científicas que estudian la actividad agraria desde una perspectiva ecológica y social (Altieri, 1987; Altieri, 1995; Gliessman, 1997; Guzmán \& Alonso, 2007). La agroecología se ha materializado a nivel mundial como una estrategia de desarrollo rural sustentable con un fuerte componente endógeno, dando lugar a numerosas experiencias de agricultura ecológicamente más sana, socialmente justa, económicamente viable y culturalmente aceptable. En Colombia, la evidencia más consistente de esta estrategia es la agricultura 
y ganadería ecológica, la cual está articulada con otras iniciativas locales, permitiendo a los productores permanecer en la actividad agraria, a la par de mejorar el estado de los recursos naturales (Alonso, 2004; Alonso, 2005). Características comunes de una buena parte de estas experiencias son la revalorización de los recursos locales tales como: materia orgánica, conocimiento de los agricultores, variedades de cultivo, semillas, razas ganaderas criollas y paisaje, la articulación con otras actividades económicas como el ecoturismo, la educación ambiental y el desarrollo de canales de comercialización cortos que permiten a los productores la captación de un mayor valor agregado.

De esta manera se evidencian dos (2) modelos de agricultura: uno tradicional, derivado de la revolución verde de los años 60 (Altieri, 1995), altamente dependiente de insumos agroquímicos, y otra agroecológica o alternativa, donde se hace uso de los recursos existentes y hay una alta rotación y uso de la energía para la producción de alimentos.

Sin embargo, la transición del modelo "convencional" al modelo "agroecológico" no es una ruta cómoda. Los productores identifican numerosas dificultades que en mayor o menor grado les afectan. Esta transición es un proceso complejo en el que se articulan distintas escalas desde la finca, el sistema local y los sistemas globales, y se ven afectadas por factores sociales, económicos, tecnológicos, culturales y ecológicos.

Desde el punto de vista ecológico, la función del grado de especialización e intensificación que tengan en la finca requerirán mayores o menores esfuerzos para eliminar los productos agroquímicos de síntesis, reordenar los flujos de nutrientes y energía, reintroducir biodiversidad como: cercas vivas, rotaciones, policultivos y sistemas ganaderos sostenibles, para disminuir el peso específico de empresas individuales. El apoyo gubernamental en lo que tiene que ver con situación financiera de los predios, tiene gran importancia en la posibilidad de acceder a créditos con bajas tasas de interés, pero que estos, a su vez, están re direccionados hacia la financiación de un modelo agroecológico convencional, con alto grado de utilización de agroquímicos. Por lo tanto, la existencia de estructuras de apoyo, como servicios accesibles de asistencia técnica y comercial, asociaciones de consumidores, organizaciones de agricultores y grupo social que lo conforma, favorece activamente la transformación, siendo esta determinante para lograr cambios en el manejo de la producción. Tecnológicamente, la capacidad de los agricultores para generar tecnologías adaptadas a las condiciones locales y de la producción ecológica, acelera o retrasa la incorporación a la agroecología.

Existe obstáculos que dificultan la transición agroecológica de los agricultores en Colombia y esto depende de dos aspectos: el primero, que no hay una investigación suficiente y sistemática desde las instituciones públicas o desde las iniciativas privadas que inviertan en la trabajos para mejorar la gestión social (Altieri, 1995), para que éstas sean productivas y con rentabilidad económica, así como en los stocks de capital humano, no están capacitados, ni hay una política firme de educación rural para la producción de productos ecológicamente limpios y de economía solidaria en lo social y en lo colectivo. Y el segundo, tal vez el más importante, tiene que ver con los espacios participativos de construcción de desarrollo endógeno, el cual en el ámbito de la agroecología se conoce como Metodologías Participativas. No se puede seguir haciendo replicación de modelos y características productivas sin realizar una base de trabajo participativo en el cual, cada campesino, cada productor, tenga un aporte a la agricultura ecológica, presentado sus experiencias exitosas, pero sin que estas sean recetas de aplicación universal.

\section{Factores que limitan la transición hacia un ma- nejo ecológico en la agricultura y la ganadería.}

A continuación se describen las más importantes barreras que dificultan que los procesos de transición agroecológica se logren: 
1) Barreras técnicas. Los bajos niveles de escolaridad de campesinos hacen que haya desconocimiento de propuestas técnicas en la agricultura y la ganadería ecológica; también hay escasez de información y conocimientos sobre experiencias para aplicar a la agroecológica en condiciones específicas. La capacitación gubernamental a cargo del SENA y las UMATAS, se centra en los monocultivos y cultivos de producción industrial, desconociendo la producción de alimentos como eje fundamental del desarrollo de comunidad. El modelo productivo de los pobladores, está basado en el consumo de agro tóxicos para la producción agrícola. Olvidando la experimentación en la producción de alimentos con los modelos agroecológicos y de auto sostenimiento.

2) Barreras sociales. La sociedad de los productores y las presiones del entorno social para abortar el proceso de cambio. Falta de apoyo desde estructuras organizativas propias de los agricultores y ganaderos, organizaciones de productores, y cooperativas. Los mayores requerimientos en la agricultura ecológica es la mano de obra, que a su vez puede actuar como bondad del modelo. Esta circunstancia, sin embargo, podría beneficiar a la mal trecha agricultura familiar.

3) Barreras de mercado y/o económica. Se identifican también, dificultades en la comercialización en el mercado interno. La escasa demanda del mercado interno de alimentos ecológicos tiene mucho que ver con la dificultad de acceso a estos productos, escasos puntos de venta y con los precios especulativos que muchas cadenas comerciales mantienen para estos productos. Los precios que no corresponden, ni con los costos de producción, ni con los precios pagados a los agricultores.

4) Barreras legales. La desprotección del productor ecológico en relación a la contaminación difusa, tanto de sustancias químicas nocivas, como de genes provenientes de organismos modificados genéticamente (OGM). La contaminación difusa está originada por los tratamientos químicos en los campos de agricultores convencionales vecinos o por otras actividades económicas más o menos cercanas, como la industria. El aire y el agua sirven de vehículo de contaminantes que aparecen después, en los exhaustivos análisis que se realizan a los productos ecológicos que deben ser desviados hacia los mercados convencionales. Esto origina una importante pérdida de ingresos para el productor ecológico. Esta situación es posible que se agrave en el futuro con la contaminación genética derivada de la presencia de cultivos transgénicos, dejando a los agricultores ecológicos en situación de indefensión ante los que contaminan.

La complejidad de las dificultades para promover la transición agroecológica, apenas esbozada en las líneas anteriores, reclama también cambios en el enfoque de la investigación que se viene realizando en Colombia.

\section{La Investigación Participativa como herra- mienta de la transición agroecológica y el desarrollo rural}

Los procesos de modernización de la agricultura a nivel mundial y la implantación de las técnicas de la Revolución Verde se llevaron a cabo con gran apoyo institucional, materializado en servicios de investigación y de extensión agraria bien dotados de recursos económicos y humanos. El modelo de investigación y transferencia de tecnología vertical y unidireccional en que se basó la modernización de la agricultura fue muy criticado a partir de los años setenta del pasado siglo (Chambers \& Ghildyal, 1985) por su incapacidad de ofrecer respuestas a la mayoría de los agricultores del mundo (los de bajos recursos y aquellos que manejaban áreas ecológicamente sensibles) y por las deficiencias intrínsecas de un modelo que incorporaba numerosos prejuicios en su seno y era incapaz de reconocer el conocimiento campesino (Cernea et al., 1985; Chambers, 1983 y 1991; Chambers \& Ghildyal, 1985). 
En líneas generales, este marco de modernización agraria, apoyado desde la investigación y extensión agrarias de carácter público, ha supuesto que los agricultores hayan tenido que realizar fuertes inversiones monetarias para la adquisición de tecnología exógena, con el fin de que sus fincas se convirtieran en eficaces unidades de obtención masiva de mercancías agrarias, incrementando su productividad física y económica. Sin embargo, en el mejor de los casos, junto a aumentos productivos han ido apareciendo una serie de externalidades negativas de orden económico, endeudamiento de los agricultores, desequilibrios de rentas entre la población urbana y rural. El despoblamiento de amplias áreas rurales, envejecimiento de la población rural y ecológica, consistente en la contaminación de recursos hídricos, erosión y pérdida de fertilidad de los suelos, deterioro de la biodiversidad, que cada vez se hacen más evidentes. Por el contrario, la transición agroecológica actual, entre otros aspectos, trata de minimizar tales externalidades negativas, se están llevando a cabo mayoritariamente sin el respaldo de la investigación pública, con el agravante de que los servicios de extensión agraria han desaparecido, siendo delegados en los servicios técnicos de empresas multinacionales, vendedores principalmente de semillas, fertilizantes y plaguicidas, a las que no conviene una transición agroecológica que vaya más allá de una sustitución de insumos.

Implementar metodologías de Diagnostico Rural Participativo (DRP) que permitan diseñar conjuntamente con los agricultores y ganaderos, propuestas de manejo y tecnologías adaptadas a sus condiciones tanto socioeconómicas como medioambientales, cuyo criterio de validez sea la capacidad de las mismas para incrementar la sustentabilidad agraria. Se entiende por DRP aquella que constituye un proceso de interacción creativa dentro de las comunidades rurales mediante el cual el conocimiento local y el científico se combinan y se desarrollan en pie de igualdad para encontrar soluciones a los problemas de los productores, sacando el máximo provecho posible de las oportunidades y recursos locales. Implica la colaboración en las tres escalas mencionadas en la introducción finca, sociedad local y sociedad mayor de agricultores e investigadores para analizar el agroecosistema. Definir los problemas y prioridades locales, experimentar con las posibles soluciones, evaluar los resultados y comunicar los hallazgos a otros agricultores y ganaderos. Posteriormente, se plantea el DRP como herramienta para solventar tales limitantes.

En el ámbito de la agroecología la propuesta del Marco para la Evaluación de Sistemas de Manejo incorporando Indicadores de Sustentabilidad (MESMIS) desarrollada por varios autores (Astier \& Hollands, 2005) ha sido la más empleada por agroecólogos con el fin de analizar los sistemas agrarios a escala de finca desde una triple perspectiva: económica, social y ecológica. El MESMIS sigue los siguientes pasos metodológicos:

1) Selección y caracterización previa del agro ecosistema mediante la obtención de la información básica, entrevista, información secundaria, para definir el itinerario técnico y la estructura y los límites del agro ecosistema.

2) Determinación de puntos críticos que amenazan la sustentabilidad del sistema y selección de indicadores correspondientes. Dichos indicadores están relacionados con todos los atributos de la sustentabilidad: productividad, estabilidad, resiliencia, equidad, autonomía y adaptabilidad cultural (Conway, 1987).

3) Definición de los criterios operativos a considerar: máximos y mínimos admisibles.

4) Medición y monitoreo de los indicadores, como por ejemplo: erosión, rendimiento, eficiencia y autonomía energética.

5) Integración de los resultados mediante análisis multi criterio y presentación de los resultados generalmente en un gráfico AMEBA.

6) Discusión de los resultados y recomendaciones. 
El seguimiento de tales pasos metodológicos permite al DRP, una rápida extracción y análisis de información referente a la estructura y funcionamiento del agro ecosistema, la consideración de la sustentabilidad como propiedad emergente a este nivel y la generación de indicadores cuantitativos y cualitativos para la evaluación de la sustentabilidad a nivel de finca. Como resultado, la información que ofrece es útil para la discusión y toma de decisiones a distintos niveles: para los agricultores, que pueden tomar medidas para mejorar la sustentabilidad; para los políticos, que tienen la posibilidad de elaborar políticas agrarias que corrijan los puntos críticos que ponen en peligro la sustentabilidad del sistema; y por último, para los investigadores, a los que ofrece la posibilidad de trabajar en grupos multidisciplinares y de obtener una información sistémica clave para reconducir sus investigaciones disciplinares hacia la resolución de los problemas que afectan a la sustentabilidad de los agro ecosistemas. Soluciones que posteriormente deben ser evaluadas igualmente desde una perspectiva sistémica y participativa, con criterios de sustentabilidad.

\section{La Investigación Diagnóstico Rural Partici- pativo (DRP): un marco metodológico para la transición agroecológica global y el Desarro- Ilo Rural Sustentable}

La Investigación DRP surge de las Ciencias Sociales en los años cuarenta del pasado siglo, aunque en el ámbito del trabajo con la población campesina, partió de un cuestionamiento a fondo de los sistemas de extensión y capacitación utilizados para la modernización del agro, que inició Paulo Freire (1978) con su obra ¿Extensión o Comunicación? La concientización en el medio rural. La IAP parte de la base de que cualquier proceso de desarrollo que se emprenda estará sesgado si no integra las realidades, necesidades, aspiraciones y creencias de los beneficiarios y más aún, si no integra a los beneficiarios de este proceso como protagonista del mismo.
De forma resumida el sociólogo Fals Borda (1991) explica que el objetivo del DRP es generar un conocimiento liberador que parte del propio conocimiento popular y que explica su realidad globalmente, enfoque sistémico, con el fin de iniciar o consolidar una estrategia de cambio, procesos de transición, paralelamente a un crecimiento del poder político, destinados ambos a alcanzar transformaciones positivas para la comunidad a nivel local; y a niveles superiores en cuanto que es capaz de conectarse con experiencias similares a redes.

El hecho de partir del conocimiento popular, en nuestro caso, del conocimiento de los agricultores, sitúa la participación de éstos en el centro del proceso de investigación. La participación de los productores, que tienen una percepción sistémica del agroecosistema, combinado con el uso de herramientas como el MESMIS, garantiza la comprensión holística de la realidad por parte del investigador. Por otra parte, la IAP se ha demostrado eficaz y tiene gran experiencia en promover cambios sociales, esto es, procesos de transición, porque es capaz de trabajar a distintos niveles: finca, sociedad local y sociedad mayor. En este sentido, intenta no sólo promover el cambio tecnológico, sino también favorecer la adquisición de capacidades y la organización por parte de los grupos implicados para que puedan continuar el proceso por sí mismos, así como incidir en ámbitos superiores (sociedad local o mayor).

\section{Metodología}

El PNN Chingaza, declarado patrimonio hídrico de la humanidad con el título de Humedales Ramsar (Sarmiento, 2007), está ubicado en la provincia del Guavio, conformada por ocho municipios: Gachalá, Gama, Guasca, Guatavita, Junín, La Calera, Ubalá y Gachetá; siendo este último, la capital de la provincia, cuenta con una población de 79.621 habitantes (DANE, 2005). El 80,1\% de sus viviendas son rurales y el 19,9\% urbanas. (Cundinamarca, 2014). Esta zona es de gran importancia para Colombia y en especial para los departamentos 
de Boyacá, Cundinamarca y Meta, debido a que su relieve montañoso (Figura 1) genera un porcentaje importante de la reservas de agua dulce que surten los acueductos de por lo menos 20 municipios y de la capital del país, Bogotá. (Cundinamarca, 2014)

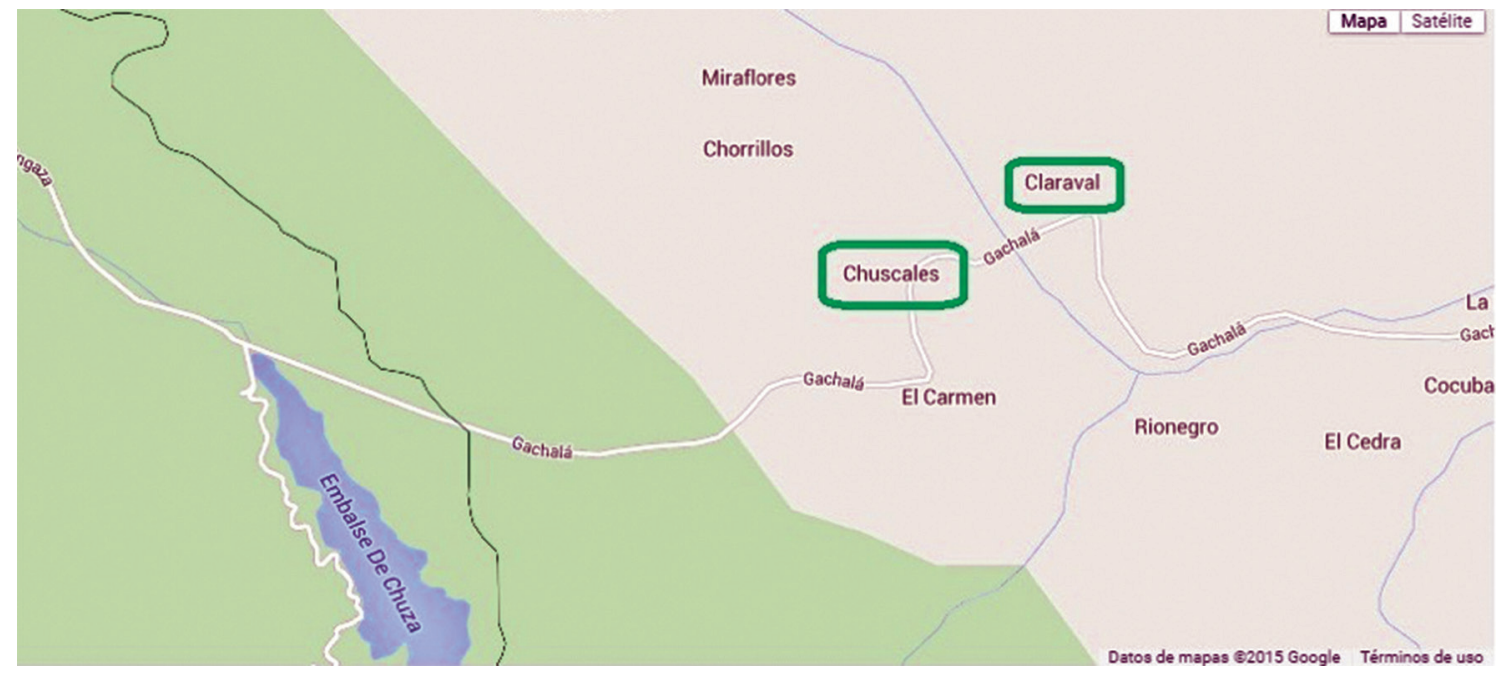

Figura 1. Mapa de ubicación de los centros poblados de Claraval y Chuscales, en relación con el parque Natural Nacional Chingaza en la región del Guavio

El proyecto de investigación, en convenio con la Corporación Universitaria Minuto de Dios, UNIMINUTO, a través del Parque Científico de Innovación Social y la Gobernación de Cundinamarca, desarrolló el plan piloto de adaptación del calentamiento global con la comunidad de los centros poblados de Chuscales y Claraval del municipio de Junín, Cundinamarca, donde se implementó la metodología de enfoque Investigación Acción Participativa (IAP) con el propósito de reflexionar sobre la realidad y los problemas existentes, para adelantar acciones que apuntan a la transformación de la realidad que se vive; de ahí la importancia de esta metodología de trabajo para promover la organización social (Fals Borda, 1991).

Acorde a esto, tanto el equipo investigativo como la comunidad, mostrarán la manera cómo las prácticas y conocimientos tradicionales de campesinos en relación con la agricultura, la silvicultura y el manejo de recursos como el agua y el suelo, aportan a la mitigación de los impactos del cambio climático. Para lograr lo anterior, se expondrá a continuación, la forma de recolección de información, la metodología usada en los talleres con agricultores de cada comunidad, y la estrategia que logró la mayor participación de los actores locales.

\section{Diagnóstico}

La metodología utiliza el mapa conceptual (Figura 2) y basándose en la Investigación Acción Participativa (IAP) o Diagnóstico Rural Participativo (D.R.P), permite hacer un reconocimiento de la zona e identificar la problemática existente regentada por la comunidad y la forma de producción actual, que está deteriorando sistemáticamente el ambiente (CCB, 2008). En primer lugar se efectuó la aplicación de talleres participativos para la identificación colectiva de los problemas. En segundo lugar, se identificó la existencia de flora y fauna nativa en la zona. En tercer lugar se precisaron los problemas generados a partir de la forma de uso de la tierra y el consecuente deterioro ambiental. $Y$ en cuarto lugar se desarrolló el trabajo de campo para mitigar el deterioro, con propuestas agroecológicas con menor impacto en el ambiente, apropiadas para el desarrollo productivo en las fincas de los participantes.

Durante la investigación se utilizaron los siguientes instrumentos: 
1. Cartografía social: para el acercamiento a la comunidad se utilizó este ejercicio, consistente en una técnica de trabajo en grupo que permite la realización de diagnósticos participativos como resultado del reconocimiento del territorio a través de distintos mecanismos como: observación, conversatorios comunitarios, acercamientos a realidades específicas, visitas de campo, entrevistas, revisión de documentos y otras ayudas que permiten un conocimiento lo más preciso posible acerca del lugar, las relaciones y las condiciones de vida de quienes habitan, frecuentan, trabajan y conocen una zona, o región, éstas se plasman en el mapa del territorio que se va a reconstruir (Piza, 2009). Basado en lo anterior, se efectuó un reconocimiento de las viviendas de los participantes, para ubicarlos en el espacio geográfico sobre un mapa de la zona, con el propósito de obtener datos sobre el uso de la tierra, esto para generar confianza, logrando acciones positivas en relación a la forma de producción agrícola y pecuaria en la comunidad y enfocarla hacia la restauración del ambiente.

2. Entrevista semi-estructurada: se completó la información con una entrevista semi-estructurada, que consistió en un diálogo profundo con los protagonistas siguiendo una ruta de conversación enfocada en el problema de investigación cuyo objetivo es el de comprender procesos gestados en una comunidad generadora de ese proceso (Hammer \& Wildavky, 1990). La discusión giró en torno a los problemas y a las particularidades de la región como zona frágil y los factores de riesgo que conllevan al actual sistema de producción, haciendo un reconocimiento general de los factores de resiliencia o las formas de adaptación de la comunidad a los cambios, los cuales les han permitido permanecer en la zona y la vulnerabilidad que esto aporta en las formas de producción y mantenimiento de la economía familiar.

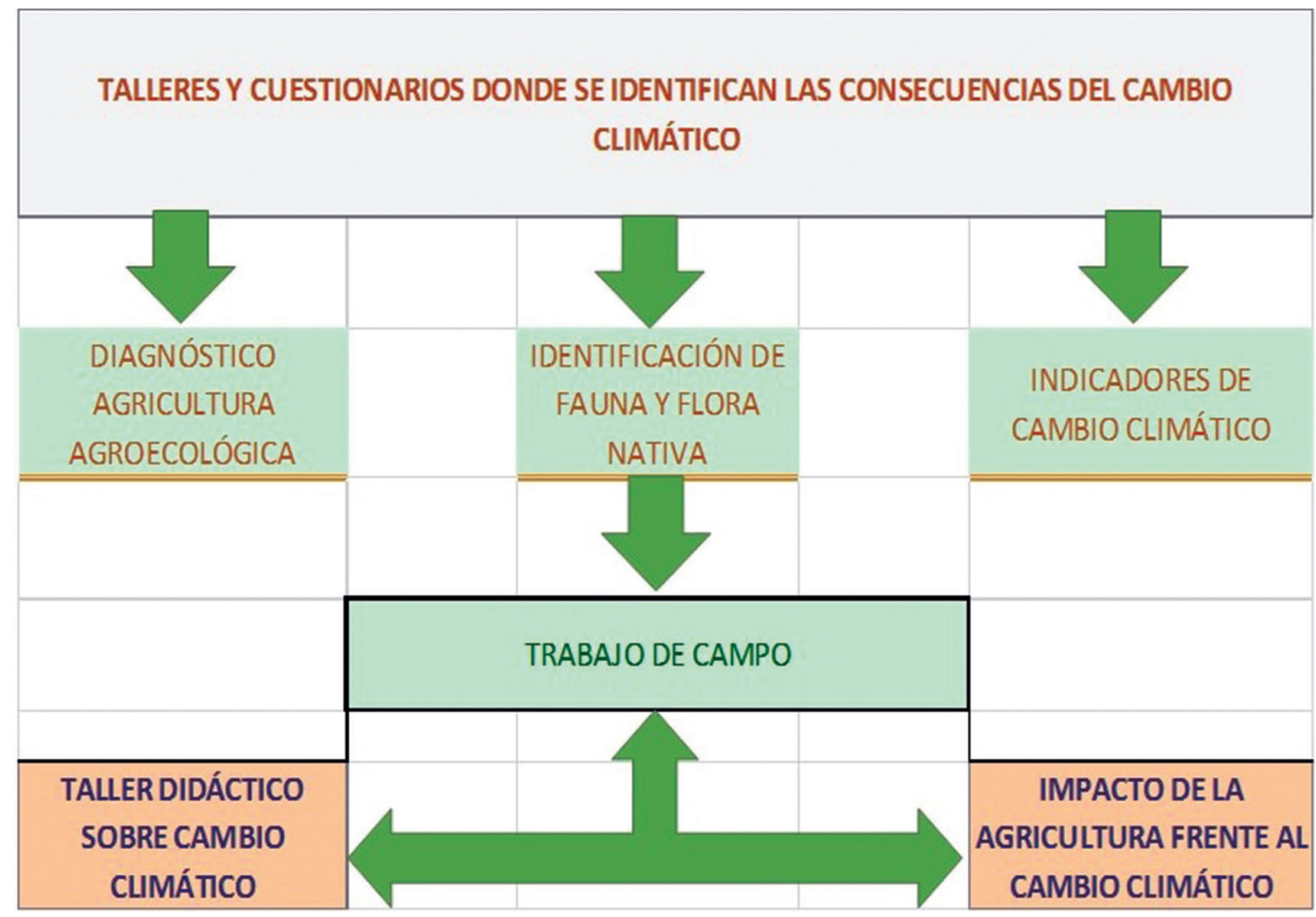

Figura 2. Mapa conceptual con el modelo de Investigación Participativa como herramienta de la transición agroecológica 


\section{Resultados}

\section{Diagnostico Rural Participativo (DRP) en la comunidad:}

Teniendo a los sujetos como actores de su propio desarrollo y no como objeto de estudio, con el trabajo de investigación realizado en los centros poblados de Chuscales y Claraval se logró que los actores sociales se empoderaran del proceso de trasformación del entorno familiar y el entorno comunitario.

En la observación participante, se concentraron los saberes y se identificaron los temores de la población frente a los cambios que todos evidenciaban sin tener una propuesta para abordar el problema. Al mismo tiempo se procuró obtener información sobre la zona e indagar sobre la visión que tienen de los aspectos positivos y de la problemática económica, ecológica y social de la producción agraria local.

En la fase de la investigación participativa se pusieron en marcha grupos de trabajo con aquellos productores de la comarca interesados en realizar la transición agroecológica.

La implementación de DRP sobre los problemas de la producción agraria en la zona, medioambientales y económicos, estableció las relaciones entre los pobladores, causa-efecto, sinergias, categorizarlos por orden de importancia, identificar soluciones, establecer orden de prioridad para poner en marcha la soluciones, asignar tareas y establecer un proceso de seguimiento del proceso de transición.

Como resultados concretos de esta fase surgieron tres tipos de propuestas:

1) La realización de estudios concretos para obtener información considerada importante para resolver la situación, tanto relativa al manejo agrícola o ganadero, fertilización, manejo de plagas o enfermedades, evalua- ción de cargas ganaderas, recuperación de variedades tradicionales y de conocimiento del manejo tradicional en la zona, como a otras barreras: de mercado, sociales, etc.

2) La realización de acciones encaminadas a la formación del grupo en determinados aspectos.

3) Acciones tendentes a transformar la situación en la que se encuentran, puesta en marcha de fórmulas asociativas, de mercados locales, de grupos de trabajo, generar redes de apoyo con otros sectores de la sociedad. Los puntos 2 y 3 corresponden en esencia a la fase siguiente de acción participativa, aunque en la realidad es difícil establecer una frontera clara entre ambas fases.

\section{La acción participativa.}

Teniendo en cuenta la estrecha línea que separa ésta de la fase anterior, es preciso resaltar que DRP concede gran importancia a la creación de redes de trabajo conjunto entre grupos sociales con similares intereses de agricultores, consumidores, técnicos. Los objetivos de estas redes son generar sinergias mediante la puesta en marcha de acciones conjuntas, optimizar el aprovechamiento de los recursos disponibles, movilizar recursos económicos, facilitar el intercambio de información, apoyar iniciativas y actuaciones decididas en el seno de las redes, y servir de foros de debate. Todo ello se encuentra dentro de esta fase.

La evaluación. Dada la complejidad de los proyectos de DRP, son posibles dos tipos complementarios de evaluación. La primera admite la verificación del conocimiento producido mediante los métodos ortodoxos de contrastación de las ciencias sociales y naturales en el caso de los estudios planteados. La segunda se basa en la evaluación de la efectividad de los cambios logrados como resultado de la acción a través del seguimiento de los indicadores propuestos como por 
ejemplo: empleo generado, porcentaje de producto vendido en canales cortos de comercialización, agricultores implicados. Esta fase de evaluación permite, tanto valorar el proceso en sí, como generar información continua para reconducirlo en caso necesario.

En definitiva la DRP, al situar en el centro del proceso de transición agroecológica a los grupos de productores, no sólo logra efectuar un diagnóstico holístico de la situación de partida que atañe tanto a la finca como a la sociedad local y mayor, y la definición de una situación objetivo realista con criterios de sustentabilidad, sino que también logra que el grupo se movilice para la consecución de las metas propuestas y que establezca relaciones con otros grupos, constituyendo redes o asociaciones que logran facilitar el cambio en distintos ámbitos, poniendo bases sólidas de desarrollo rural sustentable (Guzmán, 1998).

Los resultados obtenidos de la interacción del grupo de investigación y la comunidad campesina objeto de la intervención, están concentrados en la recuperación de pequeños lotes para la producción de hortalizas y frutas al igual que lotes de pastoreo establecidos con arreglos silvopastoriles como elemento indispensable para el mejoramiento de la alimentación del ganado.

Una vez identificados los problemas de la población campesina en la región, se pudieron establecer las líneas de acción para la mitigación de los efectos devastadores del sistema productivo desarrollado hasta el momento, como son: a. La búsqueda en la comunidad soluciones reales que se adapten a las necesidades del entorno; b. Empoderar a las mujeres, motores de las acciones humanitarias en cada uno de los predios donde se implementaron las propuestas productivas; c. Crear espacios de diálogo y entendimiento entre los integrantes de la comunidad, mujeres jóvenes personas de la tercera edad e inclusive niños como protagonistas del cambio.
De esta forma se logra conformar:

1. Huertas caseras con arreglos biodinámicas en la producción de hortalizas plantas medicinales.

2. Establecimiento de poteros de descanso y la siembra de árboles forrajeros de gran valor nutritivo para la alimentación del ganado de leche.

3. Siembra de cultivo de frijol en asocio con maíz para mejorar el suelo y aumentar la productividad por unidad de área.

4. Protección de todo nacimiento de agua que este en los predios de los participantes de la organización.

5. Cercado y siembra de árboles en las riveras de las quebradas que surten el acueducto de la zona.

6. Promoción y los productos de panadería, elaborados por las mujeres de la región.

7. Organización de la comunidad para lograr mercados ecológicos en la ciudad.

8. Creación de la asociación de productores de los poblados de Chuscales y Claraval.

9. Establecimiento de huertas en los colegios y escuelas de la región.

10. Participación activa de los jóvenes estudiantes de la región.

11. Organización y participación activa de las mujeres en la toma de decisiones concernientes a la forma de producción dentro de los predios.

\section{A modo de conclusión}

A inicios del siglo XXI, la situación de poca sustentabilidad de la agricultura tiene su origen en una compleja trama de problemas sociales, económicos y ecológicos, para lo cual, la propuesta 
de producción de alimentos de manera agroecológica se reclama como una estrategia efectiva de mejora en la sustentabilidad de la vida del campo y de las ciudades.

Para ser consecuentes con ello, los investigadores actuales requieren herramientas metodológicas de carácter sistémico que logren movilizar a la población rural en la resolución de dicha problemática, con el fin de realizar propuestas conjuntas de manejo y tecnologías adaptadas que incrementen la sustentabilidad agraria, asimismo permitan aumentar sus capacidades de crear, organizar, e incidir en el resto de la sociedad para buscar soluciones a los problemas derivados del cambio climático, con un apoyo real y contundente para el beneficio de todos.

La presente investigación es contundente en demostrar que en pequeña escala y con el trabajo sistemático de un grupo decidido de seres humanos, inquietos por lo que sucede a su alrededor puede, con pequeñas acciones hacer cambios importantes. Sólo resta recomendar que esta investigación, realizada en un pequeño poblado de la región del Guavio con consecuencias exitosas, se amplíe y se difunda en otras regiones rurales, donde se cuenta con jóvenes y adultos trabajando en el campo y en la ciudad, para que cada uno desempeñando un rol en el sistema agrosostenible, contribuya a un ambiente más sano y diverso.

\section{Agradecimientos}

Los autores agradecen a la SCTel de la Gobernación de Cundinamarca, a UNIMINUTO y su Parque Científico de Innovación Social-PCIS-, a las comunidades de las poblaciones de Claraval y Chuscales, participantes del Proyecto de "Fortalecimiento de la Capacidad de Adaptación al Cambio Climático en Territorios Productores de Agua en Bogotá y Cundinamarca 100-PS-3-13004" y al grupo de investigadores del proyecto:
Luis Eduardo Sanchez, Catherine Niño, Yulieth Prieto, Albert Hernández, Fabián Rojas y Juan Guillermo Cano.

\section{Literatura citada:}

1. Alonso, A. (2004). Impactos socioeconómicos de la agricultura ecológica. . Agricultura, Medio Ambiente y Sociedad. Serie Estudios, 156. Ministerio de Agricultura, Pesca y Alimentación. Madrid, 213-237.

2. Alonso, A. B. (2005). Desarrollo de la agricultura y ganadería ecológicas en espacios protegidos de Andalucía. Agroecología y agricultura ecológica. Progresos y problemas. Ed: RAERM. Murcia., Pp. 55-66.

3. Alonso, A. M. (2005). Productive and institutional multifunctionality: Organic farming in protected areas. Heszthely (Hungría): XXI Congress European Society for Rural Sociology, 22-27 de agosto.

4. Altieri, M. (1987). Agroecology. The Scientific Basis of Alternative Agriculture. . Boulder.: Wetsview Press.

5. Altieri, M. (1995). Agricultura y desarrollo sostenible. MAPA. Madrid, 151-203.

6. Astier, M. \& Hollands (2005). Seis experiencias agroecológicas en Latinoamérica. Madrid: Mundi-Prensa, ILEIA, ICCO y GIRA.

7. Bourdieu, P. (1998). 'Comprender', la Miseria Del Mundo. Buenos Aires: Fondo de Cultura Eeconómica.

8. CCB, C. d. (20 de 11 de 2008). Caracterización económica y empresarial de las provincias de cobertura de la CCB. El Guavio. Bogotá: Camara de Comercio de Bogotá.

9. Cernea, M. C. (1985). Building the Research ExtensionFarmer Continuum: Some Current Issues. The World Bank, Pp.310.

10. Chambers, R. (1983). Putting the Last First. . Longman Scientific and Technical; Harlow, Gran Bretaña, 235.

11. Chambers, R. (1991). Shortcut and Participatory Methods for Gaining Social Information for Projects. . Putting People First. Sociological Variables in Rural Development (M.M. Cernea, ed.) Oxford University Press; Washington, D.C., Chambers, R. 1991. Shortcut and Participatory Methods for Gaining Social Information for Projects. En Putting People Fi 515-537

12. Chambers, R. y. (1985). Agricultural research for resourc-epoor farmers: the farmer first and last. Agricultural Administration 20, 1-30.

13. Conway, G. (1987). The properties of agroecosystems. Agricultural Systems 24, 95-117.

14. Cundinamarca. (2014). Municipio de Junín. Recuperado de: http://www.junin-cundinamarca.gov.co/index.shtml

15. DANE. (2005). Censo Nacional Agropecuario Recuperado de: http://www.3ercensonacionalagropecuar io.gov.co/content/bolet\%C3\%ADn-t\%C3\%A9cnico10-de-noviembre 
16. Fals Borda, O. (1991). Algunos ingredientes básicos. En Acción y Conocimiento. Como romper el monopolio con investigación- acción participativa . Santafé de Bogotá.: CINEP; Pp. 7-19.

17. Gliessman, S. (1997.). Agroecology. Ecological Processes in Sustainable Agriculture. . Chelsea.: Ann Arbor Press.

18. Guzmán, G. A. (1998). Las metodoloígas participativas de investigación: un aporte al desarrollo local endógeno. Agricultura ecológica y desarrollo rural. Universidad Pública de Navarra, 25-28 de septiembre., 301-316.

19. Guzman, G., \& Alonso, A. (2007). La investigación participativa en agroecología: una herramienta para el desarrollo sustentable. Ecosistemas 16 (1), 24-36.

20. Hammer D. \& Wildavky A. (1990).....oral No. 4 Entrevista ¿Para qué?, 2361.

21. IGAC. (2009). Atlas de la distribución de la propiedad en Colombia. Cundinamarca. Bogotá D.C.: Instituto Geografico Agustín Codazzi. Recuperado de: http://www. igac.gov.co/wps/wcm/connect/d6e971004dc60fdbb948fb36b39898f6/3_la_concentraci\%C3\%B3n_ de_la_tierra_en_colombia_paginas_200-299. pdf?MŌD=AJPERES
22. Piza, H. Y. (2009). La cartografía social como instrumento metodológico en los proceso de construcción de territorio a partir de la participación ciudadana en la planeación territorial. Bogota: Pontificia Universidad Javeriana.

23. Sarmiento, E. (2007). Caracterización de sistemas productivos de la zona rural del Guavio. Ubalá: Convenio patrimonio Natural CORPOGUAVIO-EMGESA-Consevación y producción agroempresarial en la zona de influencia del Guavio.

\section{Conflicto de Intereses}

Los autores declaran no tener ningún conflicto de intereses 
